\title{
Enhanced Charge Transport in a Conjugated Polymer Blended with an Insulating Polymer
}

\section{$\operatorname{AUTHOR}(S)$ :}

Kim, Hyung Do; Iriguchi, Ryo; Fukuhara, Tomohiro; Benten, Hiroaki; Ohkita, Hideo

\section{CITATION:}

Kim, Hyung Do ... [et al]. Enhanced Charge Transport in a Conjugated Polymer Blended with an Insulating Polymer. Chemistry - An Asian Journal 2020, 15(6): 796-801

\section{ISSUE DATE:}

\section{0-03-16}

URL:

http://hdl.handle.net/2433/246283

\section{RIGHT:}

This is the peer reviewed version of the following article: H. D. Kim, R. Iriguchi, T. Fukuhara, H. Benten, H. Ohkita, Chem. Asian J. 2020, 15, 796, which has been published in final form at https://doi.org/10.1002/asia.201901743. This article may be used for non-commercial purposes in accordance with Wiley Terms and Conditions for Use of Self-Archived Versions: The full-text file will be made open to the public on 17 February 2021 in accordance with publisher's 'Terms and Conditions for Self-Archiving'.; この論文は出版社版でありません。引用の際には出版社版をご確認ご利用ください 。; This is not the published version. Please cite only the published version. 


\title{
Enhanced Charge Transport in Conjugated Polymer Blended with Insulating Polymer
}

\author{
Hyung Do Kim, ${ }^{\left[{ }^{[a]}\right.}$ Ryo Iriguchi, ${ }^{[a]}$ Tomohiro Fukuhara, ${ }^{[a]}$ Hiroaki Benten, ${ }^{[b]}$ and Hideo Ohkita*[a]
}

\begin{abstract}
Herein, hole transport in a quinoxaline-thiophene based conjugated polymer (PTQ1) mixed with an insulating polystyrene (PS) was studied by macroscopic and local current density-voltage characteristics measurements. As a result, we found that hole conductivity in PTQ1:PS blends increases as the weight ratio of PTQ1 is reduced down to $20 \mathrm{wt} \%$. This is mainly ascribed to increases in mobility because the charge carrier density would be constant in the insulating PS matrix. With decreasing PTQ1 weight ratio in the blends, the absorption bandwidth of PTQ1 and additional emission due to excimer decreased, suggesting that interchain interactions are suppressed. By measuring temperature dependent conductivity, we also found that the activation energy for hole conductivity is smaller in PTQ1:PS blends than in PTQ1 neat films. These findings suggest that trap sites decrease because of the suppressed interaction between PTQ1 chains in blend films. We also measured conductive atomic force microscope images of the blend films to clarify the local conductive property. For PTQ1 neat films, a low conductive image was observed over the entire film. For PTQ1:PS blends, on the other hand, many highly conductive spots were locally found. We thus conclude that the dilution of PTQ1 chains in the PS matrix leads to less formation of trap sites, resulting in more conductive transport in PTQ1:PS blends than in PTQ1 neat films.
\end{abstract}

Conjugated polymers have attracted continuing attention for many years as one of the most promising materials for optoelectronic devices, including organic thin-film transistors, organic light-emitting diodes, and organic photovoltaics. This is because of their unique properties, inclusive of excellent light harvesting and emission properties, and a high electrical conductivity. ${ }^{1-4}$ Among them, the electrical conductivity plays a key role in common for these three devices. It is therefore of fundamental importance to understand the charge transport property in active layers for highly efficient optoelectronic devices.

Until now, tremendous research efforts have been predominantly devoted to improvements in charge transport in conjugated polymer neat films in terms of crystallinity or molecular packing orientation.5,6 On the other hand, it is important to understand charge transport in blend films for organic photovoltaics because the active layer is typically based on a blend of an electron donor (D) and an electron acceptor (A) material for highly efficient devices. Charge transport in blends is more complicated than that in conjugated polymer neat films

[a] Dr. H. D. Kim, R. Iriguchi, T. Fukuhara, Prof. H. Ohkita Department of Polymer Chemistry, Graduate School of Engineering Kyoto University

Katsura, Nishikyo-ku, Kyoto 615-8510, Japan

E-mail: ohkita@photo.polym.kyoto-u.ac.jp

[b] Dr Hiroaki Benten

Division of Materials Science, Graduate School of Materials Science Nara Institute of Science and Technology

Takayama, Ikoma, Nara 630-0192, Japan

Supporting information for this article is given via a link at the end of the document.((Please delete this text if not appropriate)) as reported previously. For example, Blom et al. found that hole mobility in poly[2-methoxy-5-(3,7-dimethyloctyloxy)-1,4 phenylenevinylene] (MDMO-PPV) is much higher in blend films with $[6,6]$-phenyl- $\mathrm{C}_{61}$-butyric acid methyl ester $\left(\mathrm{PC}_{61} \mathrm{BM}\right)$ than that in the neat film. They attributed the increase in the hole mobility to hindered ring-like molecular conformations of MDMOPPV chains induced by $\mathrm{PC}_{61} \mathrm{BM}$ addition, which give rise to a reduced hopping distance of adjacent molecular and/or lower disorder energy. ${ }^{7}$ Nelson et al. studied charge carrier transpor in MDMO-PPV:PC ${ }_{61} B M$ blend films systematically. They found that not only electron but also hole mobility increases with increasing weight fractions of $\mathrm{PC}_{61} \mathrm{BM}$ although the weight fraction of MDMO-PPV decreases. ${ }^{8}$ We also studied photogenerated charge carriers in MDMO-PPV:PC ${ }_{61} B M$ blend films by transient absorption spectroscopy. Interestingly, we found that $\mathrm{PC}_{61} \mathrm{BM}$ radical cation is formed even for the blend films with a high concentration of $\mathrm{PC}_{61} \mathrm{BM}$ more than $30 \mathrm{wt} \%$. This is possibly due to partial hole transfer from MDMO-PPV to $\mathrm{PC}_{61} \mathrm{BM}$ domains and/or possibly due to direct or indirect photoexcitation of intermolecular charge transfer (CT) transitions of $\mathrm{PC}_{61} \mathrm{BM}^{9}$

Very recently, several groups including ours have reported the improvement in charge transport for ternary blend polymer solar cells based on D:D:A or D:A:A compared with D:A binary counterparts. ${ }^{10-16}$ More specifically, a fill factor, which is a good measure of charge transport in solar cells, is distinctively higher in ternary blend cells than in binary blend cells. This improvement would be ascribed to more balanced charge carrier mobility and/or more ordered structure of polymer chains in blend films. However, little is known about the origin of the improved charge transport, which should be understood for further improvements in fill factor and hence device performance. For an in-depth understanding of charge transport in blend films, it is required to clarify the relationship between macroscopic and microscopic charge transport properties, which is strongly dependent upon phase-separated microstructures in D:A blend films. Therefore, conductive atomic force microscopy (C-AFM) is a very powerful tool for obtaining a local conductive current map in the direction normal to the substrate with a high spatial resolution, which is directly related to charge transport network structures in blend films. ${ }^{17,18}$

Here, we studied the hole transport properties in a conjugated donor polymer blended with i) an electron acceptor or ii) an insulating polymer. As shown in Figures $1 \mathrm{a}-\mathrm{c}$, we employed a poly[2,3-bis(3-octyloxyphenyl)quinoxaline-5,8-diylalt-thiophene-2,5-diyl] (PTQ1) as a donor polymer and a fullerene derivative ( $\left.\mathrm{PC}_{61} \mathrm{BM}\right)$ as an acceptor material, and a polystyrene (PS) as an insulating polymer. We measured hole conductivity in PTQ1 neat films and both PTQ1:PC ${ }_{61} B M$ blend and PTQ1:PS blend films with different PTQ1 weight fractions As a result, we found that hole mobility increases in blend films rather than in neat films. In order to discuss the origin of the improved hole mobility, we also measured absorption, photoluminescence (PL), and time-resolved PL (TRPL) spectra and C-AFM images of these blend films. 
a)

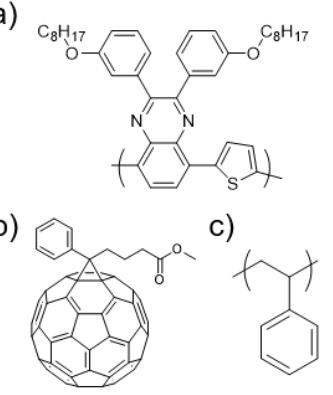

d)

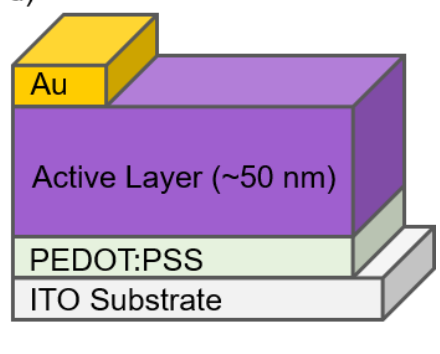

Figure 1. Chemical structures of materials employed in this work: a) PTQ1, b) $\mathrm{PC}_{61} \mathrm{BM}$, and c) PS. d) Device structure for hole-only current measurements with a layered structure of ITO/PEDOT:PSS/Active layer/Au.

In order to evaluate the hole mobility in both blend films, we measured macroscopic current density-voltage $(J-V)$ characteristics of hole-only devices with a layered structure of ITO/PEDOT:PSS/Active layer/Au as shown in Figure 1d. As shown in Figure S1, the work functions of the electrodes are similar to the energy level of highest occupied molecular orbital of PTQ1, which would ensure efficient hole collection at the junctions. Note that macroscopic $J-V$ characteristics were measured in a nitrogen-filled chamber to exclude an unintentional oxygen doping. ${ }^{19}$ As shown in Figure S2, two characteristic regions were observed: the Ohmic region with a slope of unity was observed at low voltages and the spacecharge limited current (SCLC) region with a slope of two was observed at higher voltages. The Ohmic region observed indicates the Ohmic contact at the junctions to electrodes.

According to the Ohm's law, the $J$ showed a linear correlation with the $V$ in the low voltage regime, which is given by

$$
J=\sigma V / L
$$

where $\sigma$ is the electrical conductivity and $L$ is the thickness of active layers. On the other hand, SCLC of devices can be observed in the high voltage regime in which $J$ is proportional to the square of $V$. If the trap-filled limit region due to disorders and impurities of materials is negligible, it is simply expressed by the Mott-Gurney equation as follows.

$$
J=9 / 8 \varepsilon_{r} \varepsilon_{0} \mu V^{2} / L^{3}
$$

where $\varepsilon_{\mathrm{r}}$ is the relative dielectric constant of materials, $\varepsilon_{0}$ is the vacuum permittivity, and $\mu$ is charge carrier mobility (hole mobility in this study). Here, $\varepsilon_{r}$ was assumed to be 3.5. We can then readily estimate $\mu$ by fitting the $J-V$ characteristics obtained from the SCLC measurement with the equation 2. On the other hand, $\sigma$ is given by the product of the charge carrier density $n$, the elementary charge $e$, and $\mu(\sigma=n e \mu)$. We therefore estimated $n$ by using $\sigma$ and $\mu$ obtained from the $J-V$ characteristics in the low and high voltage regions, respectively.

Figure 2 shows the apparent hole mobility $\left(\mu_{\text {app }}\right)$, conductivity $\left(\sigma_{\text {app }}\right)$, and hole carrier density $\left(n_{\text {app }}\right)$ in PTQ1:PC 61 BM blend films plotted against the weight fraction of PTQ1. With decreasing PTQ1 weight fractions, as shown in the figure, $\sigma_{\text {app }}$

increased from $10^{-7}$ to $10^{-6} \mathrm{~S} \mathrm{~cm}^{-1}$ at $50 \mathrm{wt} \%$ of PTQ1, and then decreased at $20 \mathrm{wt} \%$ of PTQ1. In other words, the $\sigma_{\text {app }}$ is one order of magnitude higher in PTQ1:PC $\mathrm{P}_{61} \mathrm{BM}(50 \mathrm{wt} \%)$ blend films than in PTQ1 neat films. On the other hand, $\mu_{\text {app }}$ also slightly increased with decreasing PTQ1 weight fraction. We note that an improvement in $\sigma$ and $\mu$ in PTQ1 domains would be more effective because weight fraction of the hole-transporting material PTQ1 is as small as $50 \mathrm{wt} \%$. On the basis of $\sigma_{\text {app }}$ and $\mu_{\text {app }}$ evaluated, we can estimate $n_{\text {app }}$ in these blend films. As a result, the trend observed for $n_{\text {app }}$ is consistent with that observed for $\sigma_{\text {app }}$, indicating that $\sigma_{\text {app }}$ is dependent primarily upon $n_{\text {app }}$ and partly upon $\mu_{\text {app. }}$. Furthermore, we found that $n_{\text {app }}$ exhibits the maximum for blend films with $50 \mathrm{wt} \%$ of PTQ1. This finding suggests that charge carriers might be partly generated from CT complex at the interface between the PTQ1 and $\mathrm{PC}_{61} \mathrm{BM}$ domains. However, this is not the case as mentioned below.

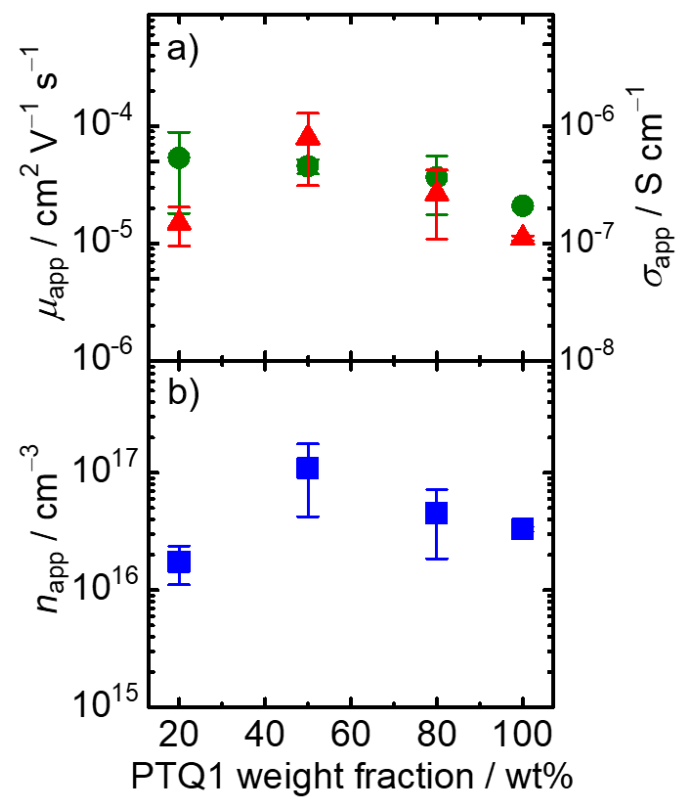

Figure 2. a) Apparent hole mobility (green circles) and conductivity (red triangles) and b) hole carrier density (blue squares) in PTQ1:PC ${ }_{61} \mathrm{BM}$ blend films as a function of PTQ1 weight fraction. The error bars for conductivity and mobility data denote the distribution in values measured for 3 and 5 devices, respectively.

We next focused on the hole mobility of PTQ1 mixed with an insulating PS to exclude the change in $n_{\text {app }}$ depending on PTQ1 weight fractions. As shown in Figure $3 \mathrm{a}$, the $\sigma_{\mathrm{app}}$ was improved with decreasing PTQ1 weight fraction from 100 to $20 \mathrm{wt} \%$. As a result, the $\sigma_{\text {app }}$ is one order of magnitude higher in PTQ1:PS (20 wt\%) blend films than in PTQ1 neat films. Similarly, as shown in the figure, $\mu_{\text {app }}$ also increased with decreasing PTQ1 weight fraction. This trend is almost the same as that observed for $\sigma_{\text {app, }}$, suggesting that $\sigma_{\text {app }}$ is mainly dependent upon $\mu_{\text {app }}$ in PTQ1:PS blend films. Considering weight fractions of PTQ1 as mentioned 
before, this finding indicates that there is a considerable increase in $\sigma$ and $\mu$ of PTQ1 domains. Indeed, as shown in Figure $3 \mathrm{~b}$, almost no change in $n_{\text {app }}$ was observed regardless of PTQ1 fractions. The order of $n_{\text {app }}$ was almost similar to that observed for PTQ1:PCBM blend films, suggesting negligible charge generation from the CT complex formed at the interface both in PTQ1:PS and PTQ1:PCBM blend films. We therefore conclude that an improvement in $\sigma_{\text {app }}$ for PTQ1:PS blends is mainly due to enhanced $\mu_{\text {app }}$, which may stem from a difference in aggregation of PTQ1 chains caused by introducing PS.

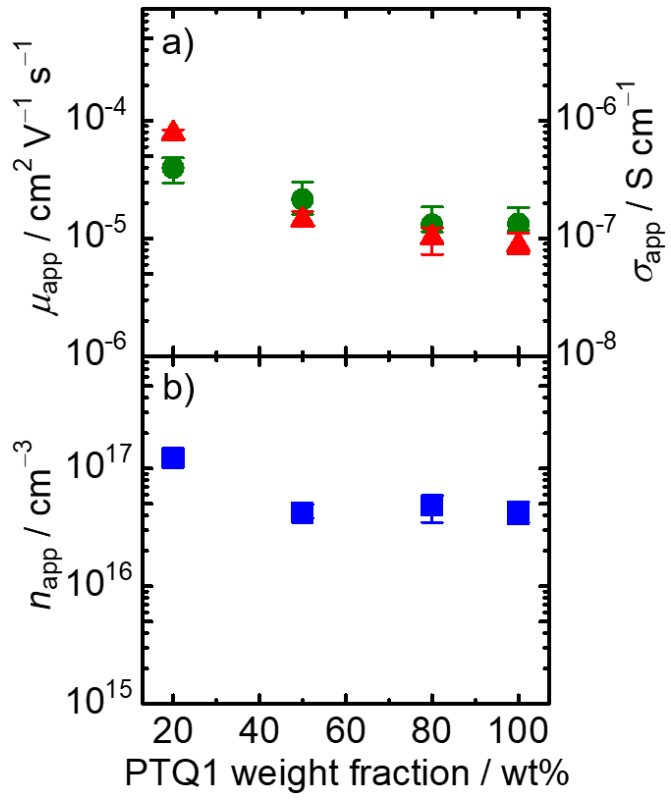

Figure 3. a) Apparent hole mobility (green circles) and conductivity (red triangles) and b) hole carrier density (blue squares) in PTQ1:PS blend films as a function of PTQ1 weight fraction. The error bars for conductivity and mobility data denote the distribution in values measured for 3 and 5 devices, respectively.

In order to discuss how PTQ1 chains are aggregated in the blends diluted by the PS matrix, we measured absorption and $\mathrm{PL}$ spectra of all the samples. Figure $4 \mathrm{a}$ shows the absorption spectra of PTQ1:PS blend films with different PTQ1 fractions. The absorption peak was red-shifted in all the films compared to that in the solution, suggesting that the effective conjugation length is longer in the film than in the solution. Interestingly, although the absorption peak was not clearly red-shifted, the absorption bandwidth increased with increasing PTQ1 weight fraction, suggesting that the energetic disorder increases most in PTQ1 neat films rather than in the PTQ1:PS blend films. As was observed with the absorption spectra, the PL peak of the films was also red-shifted compared to that in solution as shown in Figure 4b. It should be noted that a PL shoulder at around 800 $\mathrm{nm}$ becomes more distinct in the blend films as the PTQ1 weight fraction increases. This is probably ascribed to emission of excimer due to formation of $\pi-\pi$ stacking induced by interchain interactions of PTQ1. These findings suggest that with increasing PTQ1 fractions, trap sites like excimers are more likely to be formed and consequently energetic disorder increases. Conversely, trap sites and/or energetic disorder would be reduced in PTQ1 diluted with the PS matrix because of less interchain interactions.
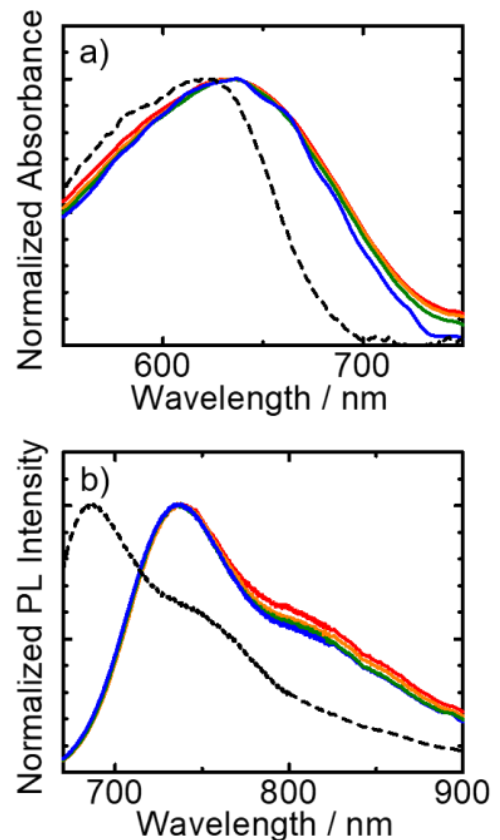

Figure 4. a) Absorption and b) PL spectra of PTQ1:PS blend films with different PTQ1 fractions: 100 wt $\%$ (red), $80 \mathrm{wt} \%$ (orange), $50 \mathrm{wt} \%$ (green), and $20 \mathrm{wt} \%$ (blue). The black broken lines in the panels a and $b$ represent absorption and PL spectra of PTQ1 in chloroform solution, respectively. For the PL measurement, the samples were excited at $640 \mathrm{~nm}$.

To better understand the origin of such a shoulder in the PL spectra, we measured TRPL spectra of PTQ1 neat and PTQ1:PS (20 wt\%) blend films. For PTQ1 neat films, as shown in Figure $5 \mathrm{a}$, the $\mathrm{PL}$ peak was observed at around $735 \mathrm{~nm}$ immediately after photoexcitation, which is due to PTQ1 monomer emission. Subsequently, it was red-shifted and finally the PL intensity significantly increased at longer wavelengths up to $800 \mathrm{~nm}$ a few nanoseconds after the photoexcitation. These spectral changes are indicative of energy migration to excimerforming sites. Thus, this red-shifted and long-lived emission is ascribed to excimer emission. In other words, there are two emission species in PTQ1 neat films: i) singlet exciton and ii) excimer. On the other hand, time evolution of the spectra for PTQ1:PS blend films with 20 wt\% of PTQ1 was slightly different as shown in Figure $5 \mathrm{~b}$. The emission peak was observed at around $735 \mathrm{~nm}$ at $0 \mathrm{~ns}$ as was the case for PTQ1 neat films. Subsequently, it was slightly red-shifted but the increase in the $\mathrm{PL}$ intensity at around $800 \mathrm{~nm}$ was noticeably reduced in comparison with that observed for PTQ1 neat films. As shown in Figure S3 and Table S1, the average PL lifetime at $790 \mathrm{~nm}$ is longer in the PTQ1:PS blend films with 20 wt\% than in the PTQ1 
neat films: the fast decay fraction decreased while the slow decay fraction increased with decreasing PTQ1 weight fractions. The latter slow fraction at longer wavelength is ascribed to excimer emission of PTQ1. Thus, these findings suggest that the dilution of PTQ1 chains in the PS matrix suppresses the formation of excimer in PTQ1:PS blends than in PTQ1 neat films because of weakened interchain interactions. As reported previously, excimer-forming sites would serve as trap sites for singlet excitons. Similarly, dimer cations are likely to be formed in such excimer-forming sites and hence would serve as charge trap sites. We thus speculate that charge trap sites would be reduced in PTQ1:PS blend films and hence charge transport is more efficient than in PTQ1 neat films.

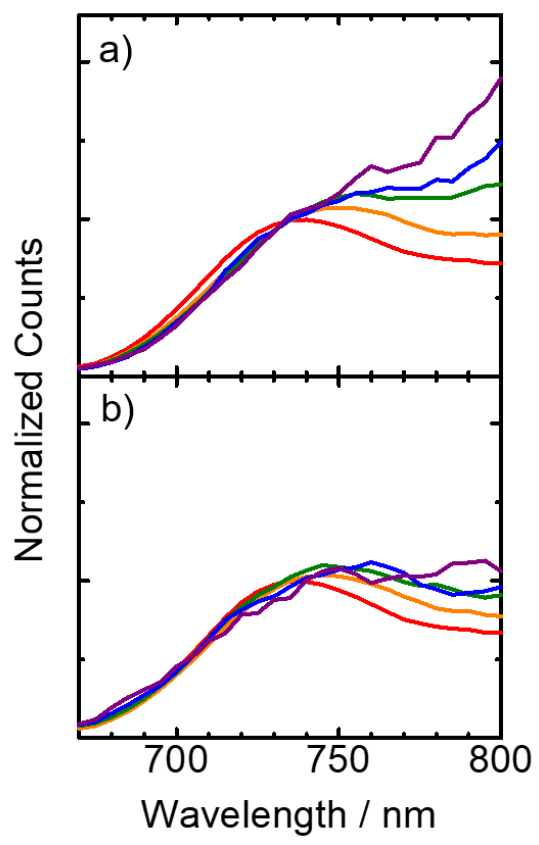

Figure 5. TRPL spectra of a) PTQ1 neat and b) PTQ1:PS blend films with 20 wt\% of PTQ1. The samples were excited at $640 \mathrm{~nm}$ and the PL spectra were monitored under different delay times: $0 \mathrm{~ns}$ (red), $1 \mathrm{~ns}$ (orange), $2 \mathrm{~ns}$ (green), 3 ns (blue), and 4 ns (purple).

We next examined how such excimer-forming sites impact on charge transport properties in detail by measuring the temperature dependence of $\mathcal{L} V$ characteristics as shown in Figure $6 a$. As a result, the $J-V$ characteristics were negligibly dependent upon temperature for PTQ1:PS blend films with 20 wt $\%$ of PTQ1 while they were clearly dependent on the temperature for PTQ1 neat films. As shown in Figure 6b, the temperature dependence of $\sigma_{\text {app }}$ can be well fitted with the Arrhenius equation, which is given by $\sigma_{\mathrm{app}}=\sigma_{0} \exp \left(-E_{\mathrm{a}} / k_{\mathrm{B}} T\right)$ where $\sigma_{0}$ is the conductivity for trap-free transport at very high temperatures, $E_{\mathrm{a}}$ is the activation energy, $k_{\mathrm{B}}$ is the Boltzmann constant, and $T$ is the temperature. From the slope, $E_{\mathrm{a}}$ was estimated to be $23 \mathrm{meV}$ for PTQ1 neat and as small as $4 \mathrm{meV}$ for PTQ1:PS blend films with $20 \mathrm{wt} \%$ of PTQ1. The smaller $E_{\mathrm{a}}$ in blend films is indicative of less charge trap sites than in PTQ1 neat films, which is in good agreement with the absorption and $\mathrm{PL}$ spectra as described above. Interestingly, there was a large difference in $y$-intersection $\left(\sigma_{0}\right)$ between them, which is a measure of trap-free charge transport at very high temperatures. This suggests that there is still a large difference in charge transport even if trap density and/or trap depth are negligible. We thus conclude that charge transport in PTQ1 chains would be improved in the blends diluted by the PS matrix.
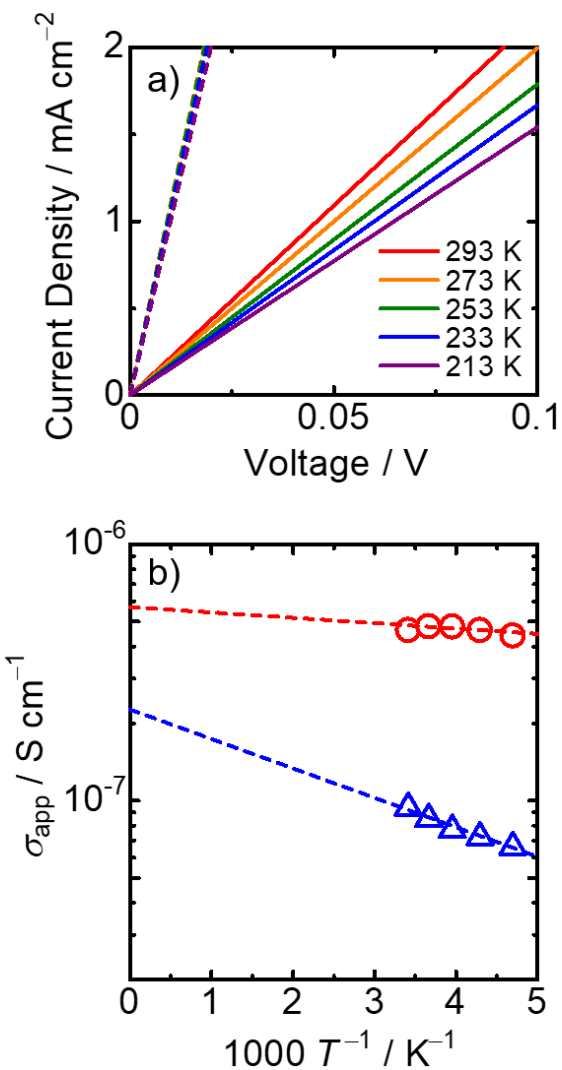

Figure 6. a) Temperature dependence of $J-V$ characteristics for PTQ1 nea (solid lines) and PTQ1:PS blend (broken lines) films with 20 wt $\%$ of PTQ1. b) Arrhenius plots of apparent conductivity $\sigma_{\text {app }}$ measured for PTQ1 neat (blue) and PTQ1:PS blend (red) films with 20 wt\% of PTQ1. The broken lines indicate fitting curves with the Arrhenius equation given by $\sigma_{\mathrm{app}}=$ $\sigma_{0} \exp \left(-E_{\mathrm{a}} / k_{\mathrm{B}} T\right)$.

We finally focus on the relationship between local charge transport properties in the direction normal to substrates and nanostructure morphology for both samples by measuring CAFM as shown in Figures $7 \mathrm{a}$ and $7 \mathrm{~b}$. We note that the thickness of samples was adjusted to be about $50 \mathrm{~nm}$ to avoid thickness dependent current density. Figures $7 \mathrm{c}-\mathrm{f}$ show the surface topographical images (c and d) and C-AFM current images (e and f) $\left(2 \times 2 \mu \mathrm{m}^{2}\right)$ for the corresponding samples. As shown in the figures, the topographical images of PTQ1:PS blend films with 20 wt\% of PTQ1 showed no clear phase-separated structures on a scale of hundreds of nanometers, which exhibit a smoother surface rather than that of PTQ1 neat films. In 
contrast, the current images of blend films clearly exhibited a substantial difference between them even with the same current scales. More specifically, there were many hot spots in the blend films where hole current was as high as up to 20 pA. In contrast, there was no hot spots: the hole current was as low as $\sim 0 \mathrm{pA}$ over the entire film for PTQ1 neat films. We also found that there is no correlation between AFM height images and CAFM current images at high current regions. This indicates that PTQ1 and PS would be well mixed together at the molecular level. On the basis of the experimental results described above, we therefore conclude that strong interchain interactions in PTQ1 neat films would induce excimer-forming sites, which are likely to serve as charge trap sites as well, resulting in a high $E_{\mathrm{a}}$ with regard to $\sigma_{\text {app }}$ and hence less efficient charge transport at room temperature. On the other hand, for PTQ1:PS blend films, the interchain interaction is effectively reduced by blending with insulating PS all together well at the molecular level, leading to less formation of trap sites, thereby providing more efficient charge transport. a)

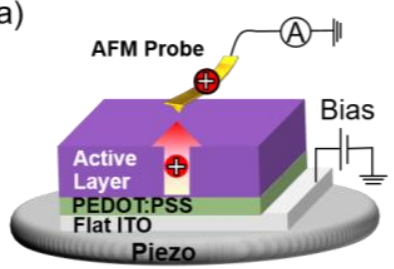

c)

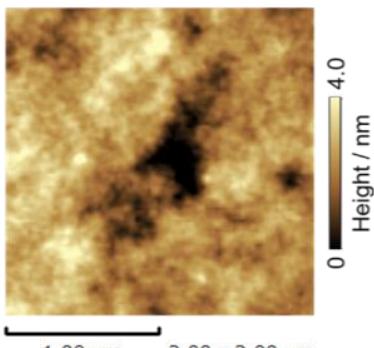

$1.00 \mu \mathrm{m} \quad 2.00 \times 2.00 \mu \mathrm{m}$

e)

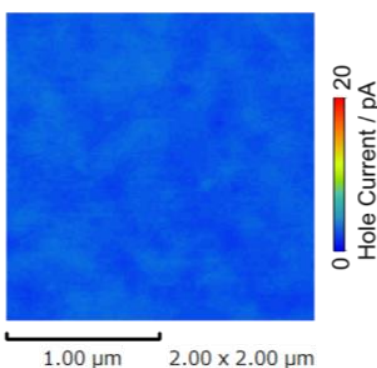

b)

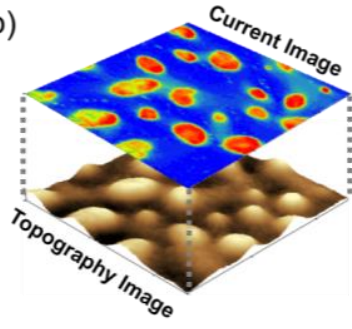

d)

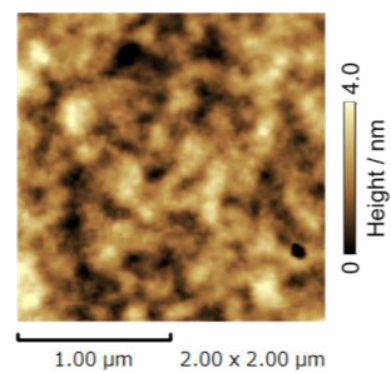

f)

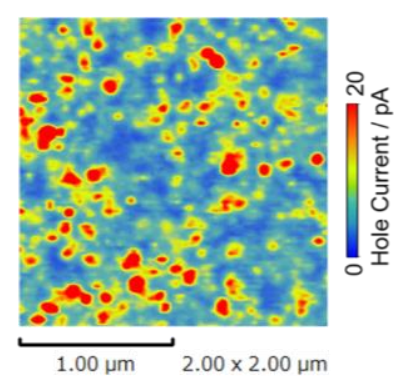

Figure 7. a) Schematic illustration of C-AFM measurement. b) Surface topographical image (bottom) and the corresponding current image (top) can be simultaneously obtained by the C-AFM measurement. Surface topographical images of c) PTQ1 neat and d) PTQ1:PS blend films with 20 wt\% of PTQ1. C-AFM current images of e) PTQ1 neat and f) PTQ1:PS blend films with 20 wt $\%$ of PTQ1.
In conclusion, we have studied the hole transport in both PTQ1 neat and PTQ1 blends mixed with i) $\mathrm{PC}_{61} \mathrm{BM}$ or ii) PS by analyzing their macroscopic and microscopic $J-V$ characteristics. As a result, we found that the hole conductivity was enhanced in PTQ1:PC ${ }_{61} B M$ blend films with decreasing PTQ1 weight fractions from 100 to $50 \mathrm{wt} \%$. This was ascribed to the increase in mobility. Similarly, it also increased in PTQ1:PS blend films with decreasing weight fraction of PTQ1 down to $20 \mathrm{wt} \%$. This is again mainly because of an increase in mobility. By measuring the absorption and PL spectra, we found the presence of a reduced shoulder in the PL spectra ascribed to the formation of excimer as well as a narrower bandwidth in the absorption spectra for PTQ1:PS blend films. This signifies less formation of excimer for diluted PTQ1 chains due to weakened interchain interactions. To study how the formation of excimer impacts on charge transport properties, we evaluated the $E_{\mathrm{a}}$ in terms of $\sigma_{\text {app }}$ by measuring the temperature dependence of macroscopic $J-V$ characteristics. As a result, it was estimated to be $23 \mathrm{meV}$ for PTQ1 neat films and to be $4 \mathrm{meV}$ for PTQ1:PS blends with $20 \mathrm{wt} \%$ of PTQ1, indicating less formation of trap sites due to suppressed excimer formation for blend films. Furthermore, we measured C-AFM to obtain local conductivity maps correlated with nanostructure morphology. For PTQ1 neat films, the hole current was as low as $\sim 0 \mathrm{pA}$ over the entire film. For PTQ1:PS blends, it was as high as up to $20 \mathrm{pA}$ at the some local spots. Such highly conductive spots would be attributed to reduced interchain interactions by mixing all together well at the molecular level. We hence believe that the dilution of PTQ1 amorphous polymers in the PS matrix results in less formation of trap sites because of weakened interchain interactions, and thereby afford more efficient charge transport. A highly efficient charge transport property has been typically achieved by using crystalline polymers. However, they would not be suitable for flexible and stretchable optoelectronic devices because of a low crack strain. ${ }^{20}$ As such, this study will evoke more interest and furthermore would offer a breakthrough towards wearable optoelectronic devices.

\section{Experimental Section}

Materials. All materials were employed without further purification as follows: PTQ1 (Solarmer Materials, Inc., $M_{\mathrm{w}}=26,750 \mathrm{~g} \mathrm{~mol}^{-1}$, and PDI = 2.14), $\mathrm{PC}_{61} \mathrm{BM}$ (Frontier Carbon, $\mathrm{E}-100 \mathrm{H}$, and $>99.9 \%$ ), and $\mathrm{PS}$ (Showa Denko, S-2.2, $M_{\mathrm{w}}=2,150 \mathrm{~g} \mathrm{~mol}^{-1}$, and PDI = 1.04)

Sample Preparation. Samples for macroscopic and microscopic $J-V$ characteristics were fabricated as follows. A hole-transporting buffer layer of poly(3,4-ethylenedioxythiophene) with poly(4-styrenesulfonate) (PEDOT:PSS, H. C. Starck Clevios PH500) was prepared atop a UV-ozone-cleaned flat ITO glass substrate by spin-coating at $3000 \mathrm{rpm}$ for $99 \mathrm{~s}$ and then dried on a hot plate at $140{ }^{\circ} \mathrm{C}$ for $10 \mathrm{~min}$. And then, active layers were prepared onto flat ITO/PEDOT:PSS substrates by spin-coating from a blend solution at $3000 \mathrm{rpm}$ for $60 \mathrm{~s}$ and then heated on a hot plate at $120{ }^{\circ} \mathrm{C}$ for $10 \mathrm{~min}$. To deposit active layer, ITO/PEDOT:PSS substrates were transferred into an inert glove box under a nitrogen atmosphere $\left(\mathrm{H}_{2} \mathrm{O}\right.$ and $\left.\mathrm{O}_{2}<1 \mathrm{ppm}\right)$. PTQ1:PC $\mathrm{P}_{61} \mathrm{BM}$ and PTQ1:PS blend solutions were prepared by dissolving the corresponding materials with a different weight fraction of PTQ1 in a chloroform solution. In the case of devices for measurement of macroscopic $J-V$ characteristics, Au electrode was additionally deposited on top of the active layer by thermal evaporation under high vacuum $\left(<2.5 \times 10^{-4} \mathrm{~Pa}\right)$. 
On the other hand, samples for absorption, PL, and TRPL spectra were spin-coated onto glass substrates in the same way as mentioned above.

Absorption and PL Spectroscopy. The absorption spectra were measured with a spectrophotometer (Hitachi, U-4100). The photoluminescence spectra were measured with a fluorophotometer (Horiba Jobin Yvon, NanoLog). The samples were excited at $640 \mathrm{~nm}$

TRPL Spectroscopy. TRPL was measured using a time-correlated single-photon-counting (TCSPC) fluorescence lifetime system (Horiba Jobin Yvon, FluoroCube). In this study, a weak excitation power ( nJ $\mathrm{cm}^{-2}$ ) was employed to avoid singlet-singlet annihilation. The samples were excited using a pulse laser with a photon energy of $1.94 \mathrm{eV}$ and a pulse duration $<200 \mathrm{ps}$ and the emission was collected from 1.85 to 1.55 $\mathrm{eV}$ after passing a long-pass filter to prevent an incoming excitation laser reflected.

Macroscopic $\boldsymbol{J}-\boldsymbol{V}$ Measurements. The macroscopic mobility measurements were performed in a nitrogen-filled chamber. On the basis of the Mott-Gurney equation, it was evaluated from $J-V$ curves in the SCLC region for hole-only devices with a layered structure of ITO/PEDOT:PSS/active layer/Au. On the other hand, the macroscopic conductivity was evaluated by four terminal techniques for accurate measurement in ambient atmosphere with an active layer of $0.25 \mathrm{~cm}^{2}$ because this method can eliminate the lead and contact resistance from the measurement. All data of conductivity and mobility were averaged from 3 and 5 devices, respectively to ensure the reproducibility of the $J-V$ characteristics.

Temperature Dependence of $\mathcal{L} \boldsymbol{V}$ Measurements. Temperature dependent $J-V$ characteristics were measured with a direct-current (DC) voltage and current source/monitor (Advantest, R6243) in a vacuum prober system (ALS Technology, VPS3-50). The temperature was controlled by the cryocooler (Twinbird Corp., SC-UF01) and monitored with a temperature controller (Panasonic, KT2).

C-AFM Measurement. C-AFM measurements were performed using a microscope (Shimadzu, SPM-9700) in contact mode with an Au-coated silicon probe (Nanosensors, PPP-CONTAu, a tip radius of $<50 \mathrm{~nm}$, and a spring constant of $0.2 \mathrm{~N} \mathrm{~m}^{-1}$ ). The surface topography and corresponding current images were simultaneously obtained with operating at a constant sample bias of $+2.0 \mathrm{~V}$. All C-AFM measurements were performed in ambient atmosphere.

\section{Acknowledgements ((optional))}

This work was partly supported by JST ALCA program Grant Number JPMJAL1404 and JSPS KAKENHI Grant Number 19K22217, Japan.

Keywords: Charge Transport $•$ Carrier Mobility $•$ Conjugated Polymer $\cdot$ Insulating Polymer $\cdot$ Blend Film

[1] C. Zhan, G. Yu, Y. Lu, L. Wang, E. Wujcik, S. Wei, J. Mater. Chem. C 2017, 5, 1569-1585.

[2] U. Zschieschang, H. Klauk, J. Mater. Chem. C 2019, 7, 5522-5533.

[3] A. Zampetti, A. Minotto, F. Cacialli, Adv. Funct. Mater. 2019, 29, 1807623_1-22.

[4] H. Fu, Z. Wang, Y. Sun, Angew. Chem. Int. Ed. 2019, 58, 4442-4453.

[5] M. Osaka, H. Benten, H. Ohkita, S. Ito, H. Ogawa, T. Kanaya, J. Phys. Chem. C 2015, 119, 24307-24314.

[6] Y. Lu, J.-Y. Wang, J. Pei, Chem. Mater. 2019, 31, 6412-6423.
[7] C. Melzer, E. J. Koop, V. D. Mihailetchi, P. W. M. Blom, Adv. Funct. Mater. 2004, 14, 865-870.

[8] S. M. Tuladhar, D. Poplavskyy, S. A. Choulis, J. R. Durrant, D. D. C. Bradley, J. Nelson, Adv. Funct. Mater. 2005, 15, 1171-1182.

[9] S. Yamamoto, J. Guo, H. Ohkita, S. Ito, Adv. Funct. Mater. 2008, 18, 2555-2562.

[10] N. Gasparini, X. Jiao, T. Heumueller, D. Baran, G. J. Matt, S. Fladischer, E. Spiecker, H. Ade, C. J. Brabec, T. Ameri, Nat. Energy 2016, 1, 16118 .

[11] H. D. Kim, R. Shimizu, H. Ohkita, Chem. Lett. 2018, 47, 1059-1062.

[12] K. Midori, T. Fukuhara, Y. Tamai, H. D. Kim, H. Ohkita, ChemPhysChem 2019, 20, 2683-2688.

[13] J. Gao, J. Wang, Q. An, X. Ma, Z. Hu, C. Xu, X. Zhang, F. Zhang, Sci. China Chem., 2020, 63, 83-91.

[14] J. Song, C. Li, L. Zhu, J. Guo, J. Xu, X. Zhang, K. Weng, K. Zhang, J. Min, X. Hao, Y. Zhang, F. Liu, Y. Sun, Adv. Mater. 2019, 31, 1905645.

[15] X. Ma, Q. An, O. A. Ibraikulov, P. Lévêque, T. Heiser, N. Leclerc, X Zhang, F. Zhang, J. Mater. Chem. A, 2020, 8, 1265-1272.

[16] M. Zhang, Z. Xiao, W. Gao, Q. Liu, K. Jin, W. Wang, Y. Mi, Q. An, X. Ma, X. Liu, C. Yang, L. Ding, F. Zhang, Adv. Energy Mater. 2018, 8, 1801968.

[17] M. Osaka, D. Mori, H. Benten, H. Ogawa, H. Ohkita, S. Ito, ACS Appl. Mater. Interfaces 2017, 9, 15615-15622.

[18] M. Osaka, H. Benten, H. Ohkita, S. Ito, Macromolecules 2017, 50 1618-1625.

[19] C.-T. Nam, J. Phys. Chem. C 2012, 116, 23951-23956.

[20] G.-J. N. Wang, A. Gasperini, Z. Bao, Adv. Electron. Mater. 2018, 4 , 1700429 . 
Entry for the Table of Contents (Please choose one layout)

Layout 1:

\section{COMMUNICATION}

Text for Table of Contents

Herein, we have studied the hole transport in a quinoxaline-thiophene based conjugated polymer (PTQ1) mixed with insulating polystyrene (PS) by analyzing macroscopic and microscopic current density-voltage characteristics. As a result, not only hole conductivity but also local current image was higher in PTQ1:PS blends with 20 wt\% of PTQ1 than in PTQ1 neat films. This is due to less formation of trap sites by dilution of

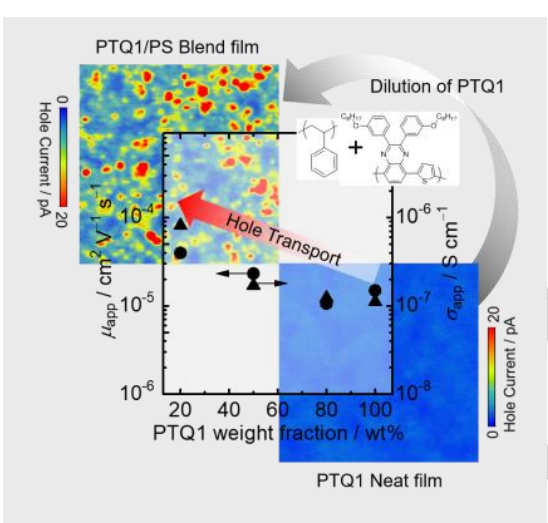

Author(s), Corresponding Author(s)*

Hyung Do Kim, ${ }^{\text {[a] }}$ Ryo Iriguchi, ${ }^{[a]}$ Tomohiro Fukuhara, ${ }^{[a]}$ Hiroaki Benten, ${ }^{[b]}$ and Hideo Ohkita ${ }^{*}$ a]

Page No. - Page No.

Title: Enhanced Charge Transport in Conjugated Polymer Blended with Insulating Polymer

Layout 2:

\section{COMMUNICATION}

((Insert TOC Graphic here))
Author(s), Corresponding Author(s)*

Page No. - Page No.

Title

Text for Table of Contents 